\title{
Planning, Design, Construction, and Testing an Experimental System to Characterize Photomultiplier Type EMI 9954B Using Cosmic Rays
}

\section{Carlos A. Cervantes Vera ${ }^{1}$, Luis Arceo, Julián Félix}

Laboratorio de Partículas Elementales, Departamento de Física, División de Ciencias e Ingenierías Campus León, Universidad de Guanajuato, México

E-mail: cervantesvc2010@licifug.ugto.mx, felix@fisica.ugto.mx,

arceodisica.ugto.mx

Photomultipliers are characteristic devices of particle detectors, used since the beginning of the $20^{\text {th }}$ century, in many experiments around the world. To use them optimally and efficiently in such detectors of broad spectrum of radiation, it is necessary to test, evaluate and characterize them. In the Laboratory of Elementary Particles of University of Guanajuato, it was planned, designed and build a twofold experimental system to evaluate and to study photomultipliers type 9954B-Emi PhotoMultiplier using cosmic rays. It has the following parts: Electronic board (which comprehends phototube feeding voltage divider and reading out analogue signal, and digitalizing circuit) and enclosure dark box (which comprises top and bottom veto counters, based each one on a $10 \mathrm{~cm}$ x $10 \mathrm{~cm}$ x $0.6 \mathrm{~cm}$ plastic scintillator and a Hamamatsu MPPC photodiode S12572-100P, and the material under study). Technical details of this evaluation system and preliminary obtained physical results are presented and discussed.

36th International Cosmic Ray Conference -ICRC2019-

July 24th - August 1st, 2019

Madison, WI, U.S.A.

\footnotetext{
${ }^{1}$ Speaker

(C) Copyright owned by the author(s) under the terms of the Creative Commons 


\section{Introduction}

Photomultipliers (PMT) are very sensible photon detectors which potential applications is currently studied by many groups in the world, in relation with their applicability in many different fields of science and technlogy, such as high-energy physics calorimetry, astrophysics, medical imaging for diagnosis, and many others [1]. A photomultiplier system is subdivided into a fototube -a photocathode, a secondary emission multiplier stages (dinodes), an anode- and an electronic-optical input system. The photoelectrons that affect the first dinode produce a series of secondary electrons which accelerate in the next dinodes of the multiplier where, in turn, they produce secondary electrons, and so on up to a measurable electrical signal on the anode.

The last dynode has the shape of a shallow box, into which the anode extends. The electrons of the penultimate dinode pass through the anode mesh to hit the last dinode. To quantify the relevant properties of PMT, a test bank has been established that allows measuring general parameters such as the number of counts vs time and analog and digital signal observations. We focus this document on planning, design, construction, and testing an experimental system to characterize photomultiplier type EMI 9954B using cosmic rays. It is based on a home made basic feeding and reading out electronic card, the EMI 9954B type photomultiplier -inside a home made Aluminium shielded black box-, two veto conters based on Hamamatsu S12572100P photodiodes -on top and bottom of the home made Aluminium shielded black box-[2].

We present the design, construction, characterization, and preliminary physical results.

\section{Planning, Design, and construction}

This experimental setup must be simple, economical, compact, and easy to handle and operate. To test one phototube at the time.

The design of the experimental system contains one home made electronic board, which is divided in five sections as follows: 1) voltage divider, for photomultiplier tube operation, a high voltage from 1200 to 2400 volts is usually applied across the cathode and anode, with a proper voltage gradient set up between the photoelectron focusing electrode, dynodes, and an accelerating electrode. 2) Passive electronic board, is the base of the RC circuit to decouple the input high voltage from the low output voltage [3], 3) Discriminator, its function is to directly compare analog signal inputs that comes from the RC circuit; with a fixed trigger voltage defined by the final user to give out the digital signal [4]. 4) Veto-detectors, the function of veto-detectors is to find a coincidence between two signals produced by cosmic rays. 5) Data acquisition System, the output digital signal is directed to a data acquisition system (DAQ, CompactRIO -cRIO-NI-9026) [5], then to a computer to storage data and to analyze it at posteriori.

\subsection{Voltage divider}

This is home made design. It is shown in Figure 1. The basic operating voltage is about $1700 \mathrm{~V}$. The final output is on TP-3 and ground. A very good output signal is obtained. 


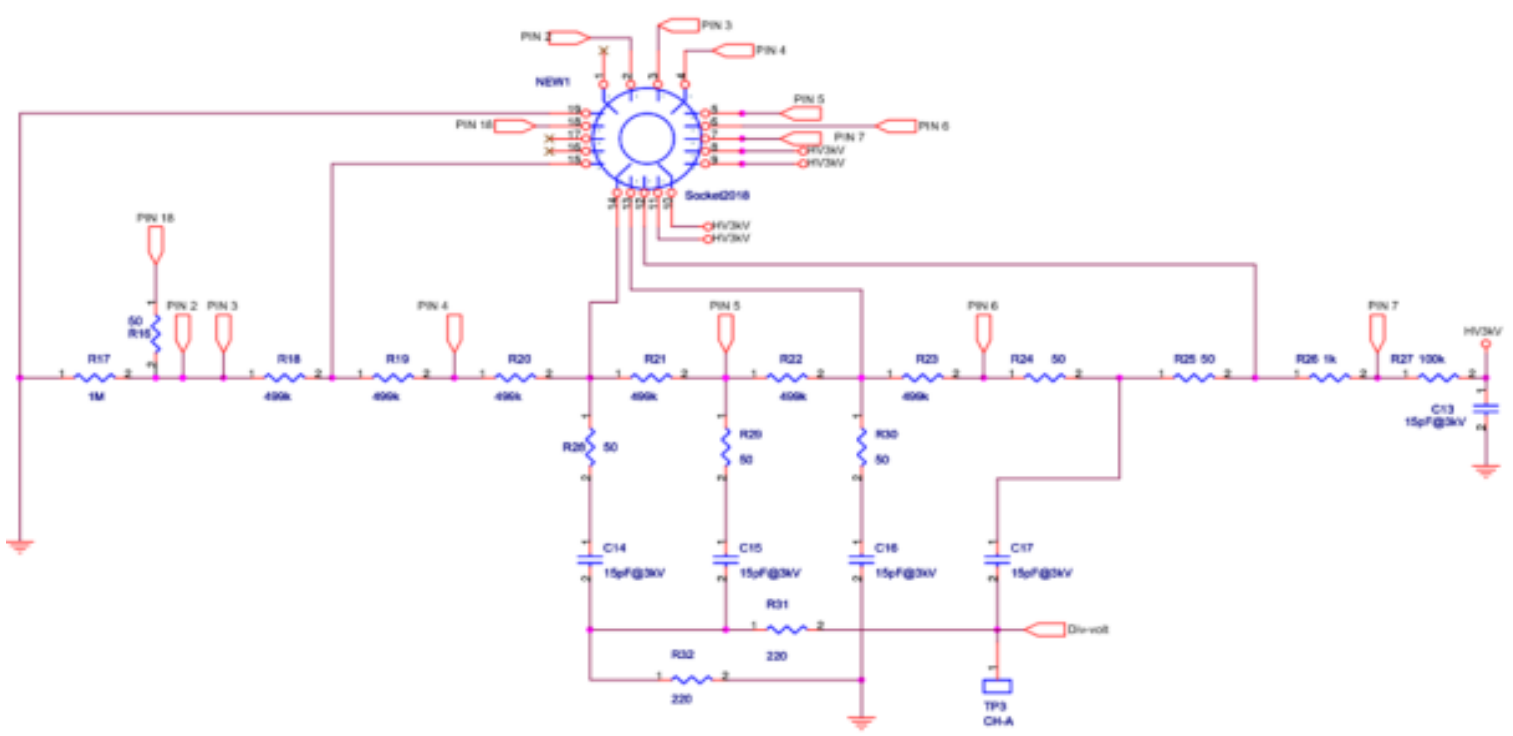

Figure 1. Voltage divider diagram.

\subsection{Discriminator board}

The electronic circuit diagram is shown in Figure 2.

The discriminator board works with ultrafast detectors. The supply voltage is $+5,-5$ volts dc. Its function is to compare the analog signal that reaches the passive electronic card and giveout a digital signal if the trigger condition is satisfied.

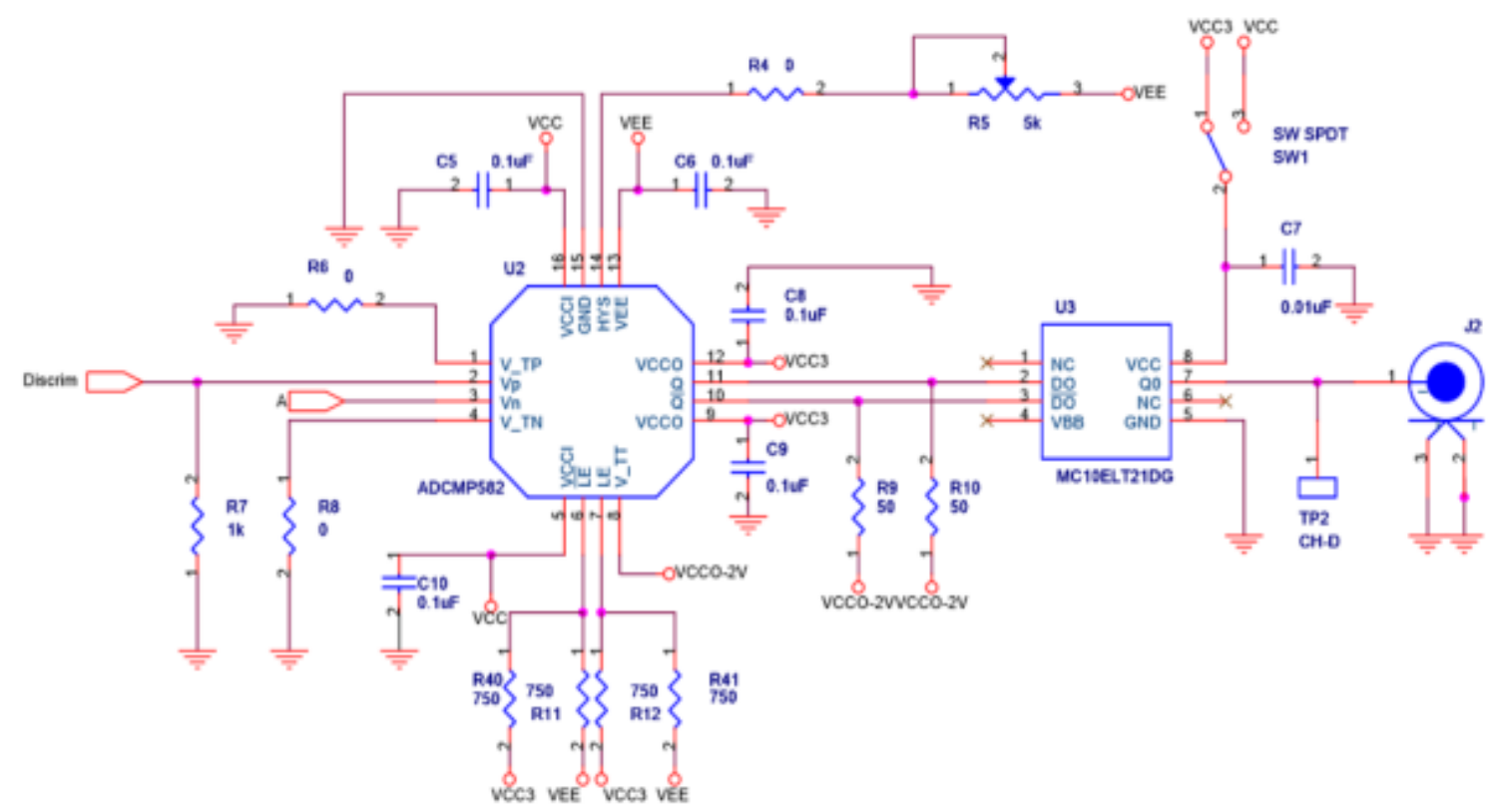

Figure 2. circuit comparator diagram

The voltage divider circuit and the discriminator circuit are located on the same electronic card, see Figure 3.1. This electronic card is positioned in a photomultiplier tube inside a ligth-tight box, Figure 3.2 


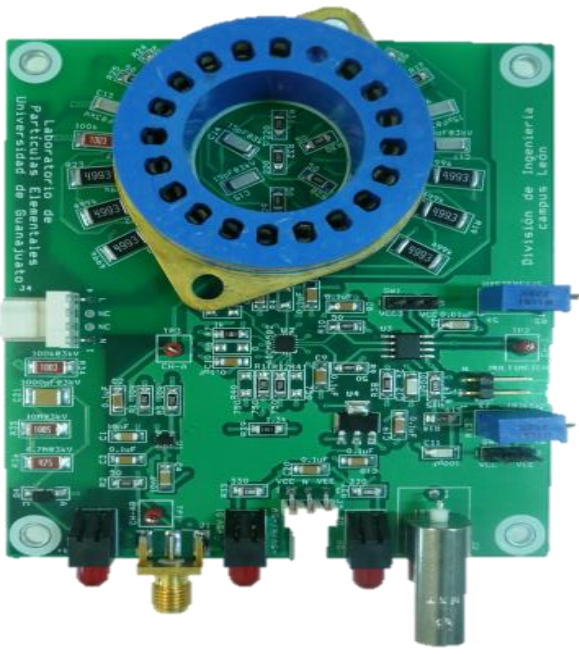

Figure 3.1. Home made electronic board.

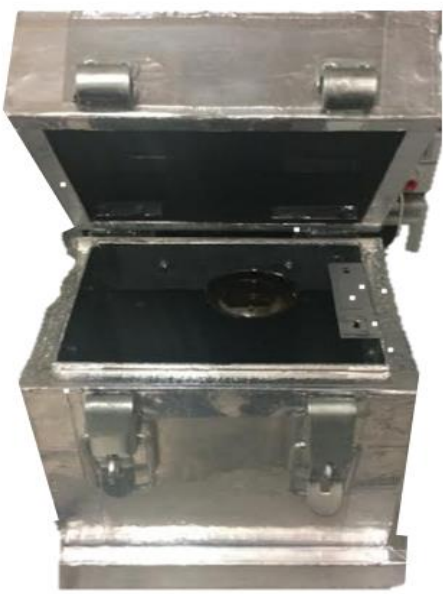

Figure3.2. Light-tight box. Black box.

\section{Characterization and Results}

The output counts as function of applied high voltage are shown in Figures 4, and 5. The recording intervals began for the first Figure at 1400 volts dc in steps of 100 volts, applied up to $2500 \mathrm{Vdc}$. For the second graph, the voltage started at 1000 volts with a range of 1000 volts up to $2500 \mathrm{dc}$ volts. These curves are characteristic of the phototubes. The plateu zone is shown about $1700 \mathrm{Vdc}$.

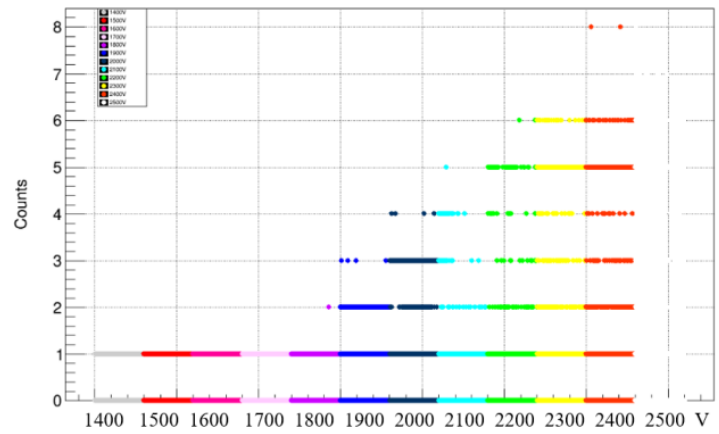

Figure 4. Counts vs HV.

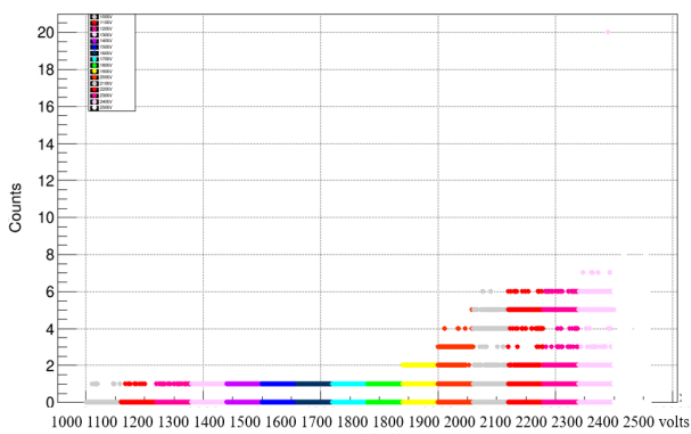

Figure 5. Counts vs HV.

\section{Conclusions}

It was designed, built, tested and characterized an experimental system to study photomultipliers EMI 9954B using cosmic rays. The dark box, the passive electronic board, the discriminating board, the data acquisition system are home made and work correctly.

\section{Acknowledgements}

CONACYT grants. México. 


\section{References}

[1] https://www.hamamatsu.com/resources/pdf/etd/PMT_handbook_v3aE.pdf. $\quad$ Flyckt, S.O. and Marmonier, C., Photomultiplier Tubes: Principles and Applications, Philips Photonics, Brive, France (2002). https://web.archive.org/web/20060908113719/http://www.jhu.edu/iic/Photomultipliers.pdf. E.Patrick, et al., Nuclear Instruments and methods. A 620 (2010)217. https://www.sciencedirect.com/science/article/pii/S0168900210008156

[2] http://www.hamamatsu.com.cn/UserFiles/DownFile/Product/s12572-025_etc_kapd1043e03.pdf Hamamatsu, datasheet, (December, 2015). http://www.hamamatsu.com/jp/en/S12572-100P.html.

[3] Luis A, J. Felix, Design, construction and characterization of a three-channel cosmic ray detector based on Aluminium blocks electronics at Tecnológico Nacional de México, Celaya, México, February 2018. Pistas Educativas, Vol. 39, Núm. 128 (2018). http://itcelaya.edu.mx/ojs/index.php/pistas/article/view/1138/0.

[4] Analog Devices, datasheet, (Rev. C, 2015-2016). http://www.analog.com/media/en/technicaldocumentation/data-sheets/AD8000.pdf .

[5] L. Arceo, J. Felix. Basic Readout Electronic Board for Three Channel Cosmic Ray Detector. The 38th International Conference on High Energy Physics 3-10 August 2016 Chicago, USA https://pos.sissa.it/282/769/pdf. 